\title{
Radiologia odontológica: Confecção de um novo dispositivo didático-pedagógico
}

\author{
Dental radiology: Making a new didactic-pedagogical device \\ Radiología dental: Fabricación de un nuevo dispositivo didáctico-pedagógico
}

Recebido: 15/07/2021 | Revisado: 21/07/2021 | Aceito: 27/07/2021 | Publicado: 03/08/2021

Ana Cristina Alves da Silva
ORCID: https://orcid.org/0000-0001-8140-9497
Universidade Federal do Pará, Brasil
E-mail: anacrisalves98@ gmail.com
Evaldo Bezerra de Oliveira
Centro Universitário Tocantinense Presidente Antorio
ORCID: https://orcid.org/0000-0003-3855-1951
Faculdade de Ciências do Tocantins, Brasil.
E-mail: evaldobo1000@ gmail.com
Fernanda Fresneda Villibor
ORCID: https://orcid.org/0000-0002-4546-7478
Centro Universitário Luterano de Palmas, Brasil
E-mail: fvillibor@ hotmail.com
Ana Lúcia Roselino Ribeiro
ORCID: https://orcid.org/0000-0003-2229-0718
E-mail: analuciaroselino@gmail.com

\section{Resumo}

O tradicional treinamento pré-clínico da radiologia em seres humanos é contraindicado, uma vez que a exposição a radiações ionizantes sem necessidade pode ser prejudicial à saúde. Atualmente, existem metodologias desenvolvidas para o ensino nas tomadas radiográficas, como uso de manequins, crânios artificiais ou até mesmo realidade virtual. No entanto, essas técnicas apresentam limitações, como não proporcionar ao acadêmico pontos de reparo anatômicos, ausência de limitação de lábios e mucosa jugal, não simulação de posicionamento adequado tanto do paciente como do aparelho de raio X, além de não acrescentar conhecimento de interpretação radiográfica e o elevado custo. Dessa forma, o objetivo desse estudo foi desenvolver uma metodologia de ensino pré-clínico prático da Radiologia Odontológica por meio de um novo dispositivo com características da arcada humana, que pode ser acoplado a um simulador de paciente, possibilitando o uso de posicionadores, posicionamento de paciente e dos feixes de raio X, além da interpretação das características e baixo custo. Para a confecção do dispositivo, utilizou-se a base de um manequim odontológico comercial, acrescido de características como dentes com câmera pulpar, trabeculado ósseo, dente tratado endodonticamente, restaurações, preparo de prótese fixa e provisório, implantes, raiz, cistos e ausência dentária. O manequim pode ser adaptado a um simulador de paciente, a fim de simular as posições de trabalho, uso de posicionadores radiográficos, posicionamento de paciente e dos feixes de raio X. Conclui-se que é possível mimetizar a cavidade bucal com baixo custo, para que o aluno adquira conhecimento de radiologia prévio à prática clínica em pacientes.

Palavras-chave: Ensino em Odontologia; Radiologia odontológica; Simulador de paciente.

\begin{abstract}
Traditional preclinical radiology training in humans is contraindicated as exposure to unnecessary ionizing radiation can be harmful to health. Currently, there are methods developed for teaching X-rays, such as the use models, artificial skulls or even virtual reality. However, these techniques have limitations, such as not having anatomical repair points and limitation of the lips and jugal mucosa, not simulating the proper positioning of the patient and the $\mathrm{X}$-ray machine, and not adding knowledge of radiographic interpretation and high cost. Thus, the aim of this study was to develop a practical preclinical dental radiology teaching method by means of a new device with characteristics of the oral cavity and teeth that can be connected to a patient phantom, enabling the use of positioners, positioning of $\mathrm{x}$-ray beams, as well as the interpretation of characteristics and low cost. To make the device, it was used a commercial dental model, added with features such as teeth with pulp camera, bone trabeculate, endodontically treated tooth, restorations, fixed prosthesis, implants, root, cysts and tooth absence. The device can be connected to a patient phantom in order to simulate working positions, use of radiographic positioners, patient positioning and $\mathrm{x}$-ray
\end{abstract}


beams. It is concluded that it is possible to mimic the oral cavity at low cost so that students can acquire knowledge of radiology prior to clinical practice in patients.

Keywords: Dentistry education; Dental radiology; Patient model.

\section{Resumen}

La formación en radiología preclínica tradicional en seres humanos está contraindicada, ya que la exposición a radiaciones ionizantes innecesarias puede ser perjudicial para la salud. Actualmente, existen metodologías desarrolladas para la enseñanza de tomas radiográficas, como el uso de maniquíes, cráneos artificiales o incluso la realidad virtual. Sin embargo, estas técnicas tienen limitaciones, como no proporcionar al alumno puntos de reparación anatómicos, no limitar la mucosa de labios y mejillas, no simular el posicionamiento adecuado tanto del paciente como de la máquina de rayos X, además de no sumar conocimientos de interpretación radiográfica y el alto costo. Así, el objetivo de este estudio fue desarrollar una metodología para la enseñanza preclínica práctica de Radiología Dental a través de un nuevo dispositivo con características del arco humano, que se pueda acoplar a un simulador de paciente, permitiendo el uso de posicionadores, posicionamiento de paciente y X -rayas de rayos, además de interpretación de características y bajo costo. Para la fabricación del dispositivo se utilizó la base de un maniquí dental comercial, sumado con características como dientes con cámara pulpar, trabeculado óseo, diente tratado endodónticamente, restauraciones, preparación de prótesis fijas y temporales, implantes, raíz, quistes y dientes perdidos. El maniquí se puede adaptar a un simulador de paciente con el fin de simular posiciones de trabajo, uso de posicionadores radiográficos, posicionamiento del paciente y haces de rayos X. Se concluye que es posible imitar la cavidad bucal a bajo costo, para que el alumno adquiere conocimientos de radiología previos a la práctica clínica en pacientes.

Palabras clave: Enseñanza en odontología; Radiología dental; Simulador de paciente.

\section{Introdução}

Em Odontologia, tomadas radiográficas fornecem informações por meio de imagens bidimensionais (2D), dos dentes e tecidos duros do complexo bucomaxilofacial (Boeddinghau \& Whyte, 2008; Ruprecht, 2009), estas são muito utilizadas como exame complementar no diagnóstico. Em contrapartida, a exposição à radiação ionizante não é isenta de riscos (Queiroz et al., 2017).

Profissionais e acadêmicos se expõem habitualmente de forma direta e indireta as radiações causadas pelo uso de raios X, aumentando sua vulnerabilidade aos riscos intrínsecos a essa exposição (ADACSA, 2006; Capelozza, 2009). Dessa forma, em âmbito acadêmico, cursos de Odontologia fazem uso de um ambiente baseado em simulação e de ferramentas que permitem praticar e desenvolver essas competências em alternativa ao uso de pacientes (Gal et al., 2011).

É fato o crescente uso de simuladores na educação em saúde, sendo bastante utilizados nas disciplinas pré-clínicas, que possuem por objetivo permitir aos estudantes a oportunidade de exercitar técnicas e ganhar experiências necessárias à prática em pacientes (Luz et al., 2015). No entanto, os manequins utilizados atualmente não suprem as necessidades para um aprendizado de forma multidisciplinar e realista.

As metodologias aplicadas atualmente apresentam limitações como não proporcionar ao acadêmico pontos de reparo anatômicos, ausência de limitação de lábios e mucosa jugal, não simulação de posicionamento adequado tanto do paciente como do aparelho de raio x, resultando em inadequada representação do ambiente clínico (Silva et al., 2018). Além disso, as simulações incluem treinamento para procedimentos clínicos usando dentes extraídos ou artificiais colocados em mandíbulas estáticas de manequim ou cabeças fantasmas (Suvinen et al., 1998; Jackson, 1993), em que é necessário um manequim para cada especialidade.

Diante disso, o objetivo deste estudo foi desenvolver um manequim odontológico com características da cavidade bucal acoplado a um simulador de paciente com o intuito de aprimorar o ensino pré-clínico de Radiologia Odontológica. 


\section{Material e Métodos}

A pesquisa foi realizada nos laboratórios de Odontologia e de Radiologia Odontológica, nas mediações do Centro Universitário Tocantinense Presidente Antônio Carlos-UNITPAC. O experimento consistiu no desenvolvimento de um dispositivo a base de um manequim com características semelhantes à cavidade bucal, possuindo componentes como dentes inclusos, restaurações, preparo de prótese fixa e provisório, implantes, trabeculado ósseo, lesões periapicais, ausências dentárias, tratamento endodôntico e perdas ósseas. Essas características foram simuladas tanto fisicamente como radiograficamente, sendo que o manequim pode ser acoplado a um simulador de paciente e adaptado à cadeira odontológica.

Para o desenvolvimento do manequim odontológico utilizou-se os seguintes materiais: manequim odontológico comercial com articulador (adaptável ao simulador de paciente), broca de Carboneto de Tungstênio Maxicut, kit acadêmico, dentes radiopacos com simulação de polpa radiolúcida, gesso tipo II, maravalha, resina composta, cimento de ionômero de vidro, amálgama, limas Kerr Flexofile, cone de guta percha, cimento endodôntico obturador sealer 26, conjunto de dois implantes, filmes radiográfico, posicionadores e colgadura. Não há marcas comerciais específicas indicadas para a confecção do novo dispositivo.

\subsection{Desenvolvimento do dispositivo}

Inicialmente foram feitos desgastes no manequim, tanto para a colocação dos dentes no espaço alveolar (Figura 1A, B e C) quanto para a colocação da mistura de gesso e maravalha e confecção de canaletas para facilitar o posicionamento do filme radiográfico (Figura $1 \mathrm{D}$ e E).

A simulação óssea é confeccionada pela mistura de gesso e maravalha, conseguida após testes de proporção, sendo estes comparados às radiografias de arcadas humanas, sendo escolhido a proporção 2:1 (40g de gesso II e 20g de maravalha triturada) (Figura1F).

Figura 1. Desgaste do espaço de colocação dos dentes (A, B e C), confecção da canaleta para colocação dos filmes radiográficos (D e E) e mistura de gesso e maravalha $(F)$.
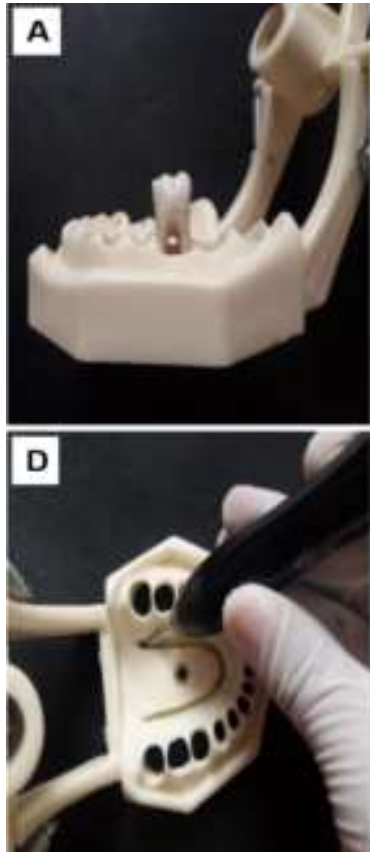
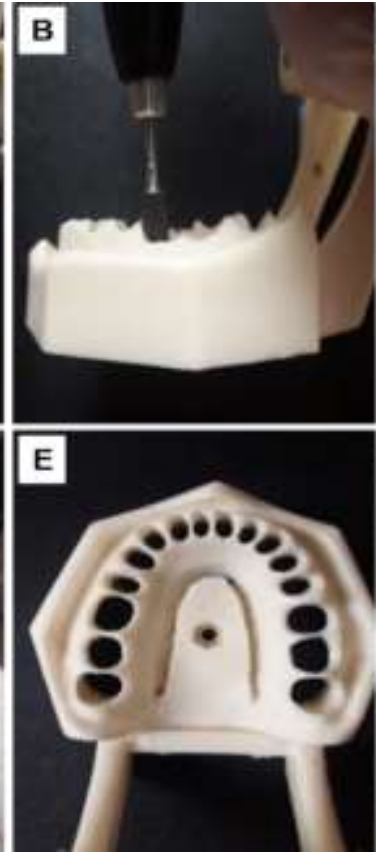

Fonte: Autoria própria.
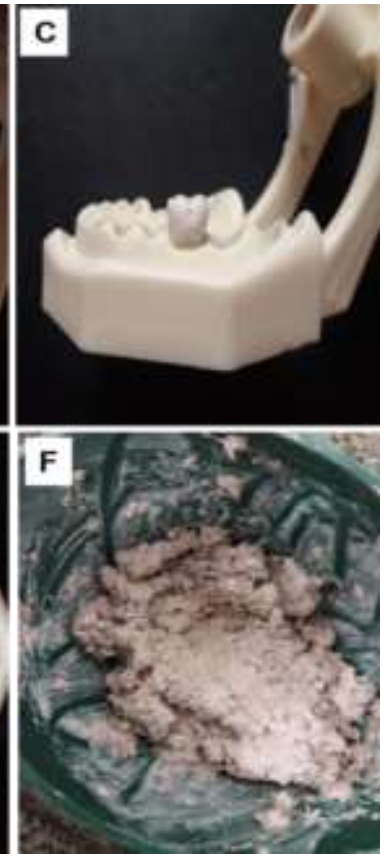$$
\text { 列 }
$$ \\ (1)}


O preparo das restaurações foi realizado nos dentes com características de radiopacidade (Figura 2A), sendo confeccionada a restauração classe I de black no dente 27 utilizando amálgama em uma cavidade rasa (Figura2B), e classe I de black no dente 26 utilizando resina composta com forramento de cimento de hidróxido de cálcio (Figura 2C). Após, realizouse radiografia dos dentes para conferir as características radiográficas (Figura 2D e E).

Figura 2. Preparo das cavidades (A), dente com restauração de amálgama (B), dente com restauração de resina composta (C), radiografia do dente restaurado com amálgama (D), radiografia do dente restaurado com resina composta (E).

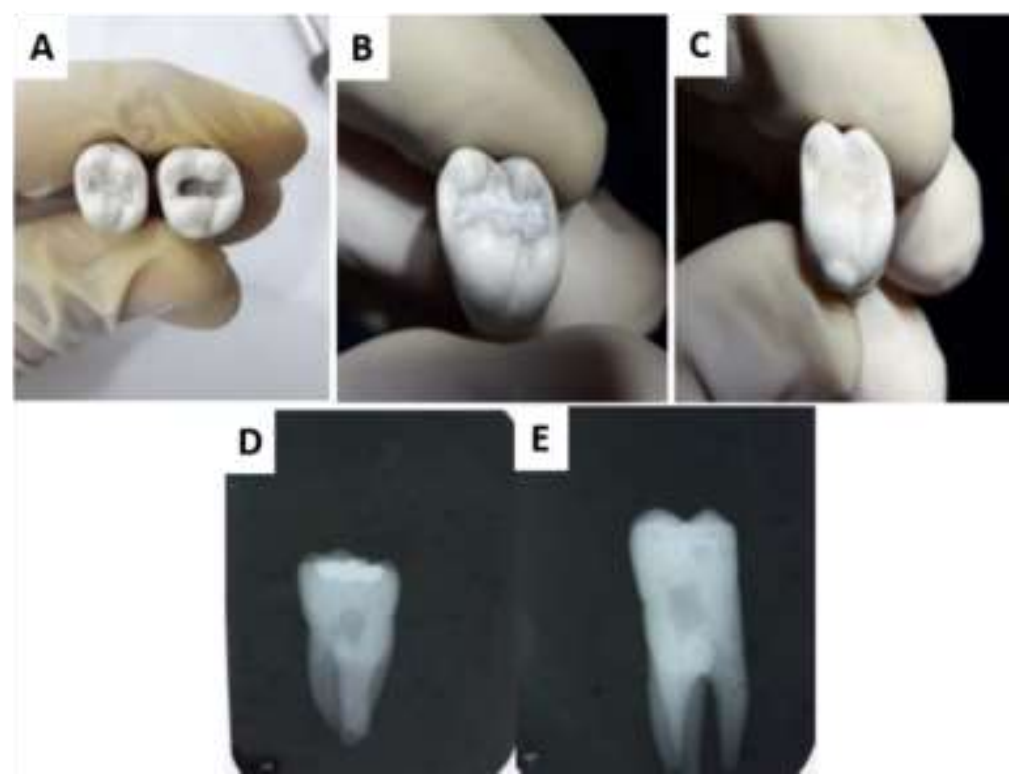

Fonte: Autoria própria.

No dente 11 foi confeccionado preparo classe IV e restauração de resina composta (Figura 3A e B). O ligamento periodontal foi confeccionado de cera 7 (Figura 3C), assim como as lesões apicais, periapicais e cisto residual. O preparo de prótese fixa e provisório foi realizado no dente 14, como visto na (Figura 3 D).

Figura 3. Restauração classe IV de resina composta (A e B), ligamento periodontal simulado com cera sobre a superfície radicular (C) e provisório do elemento 14 (D).
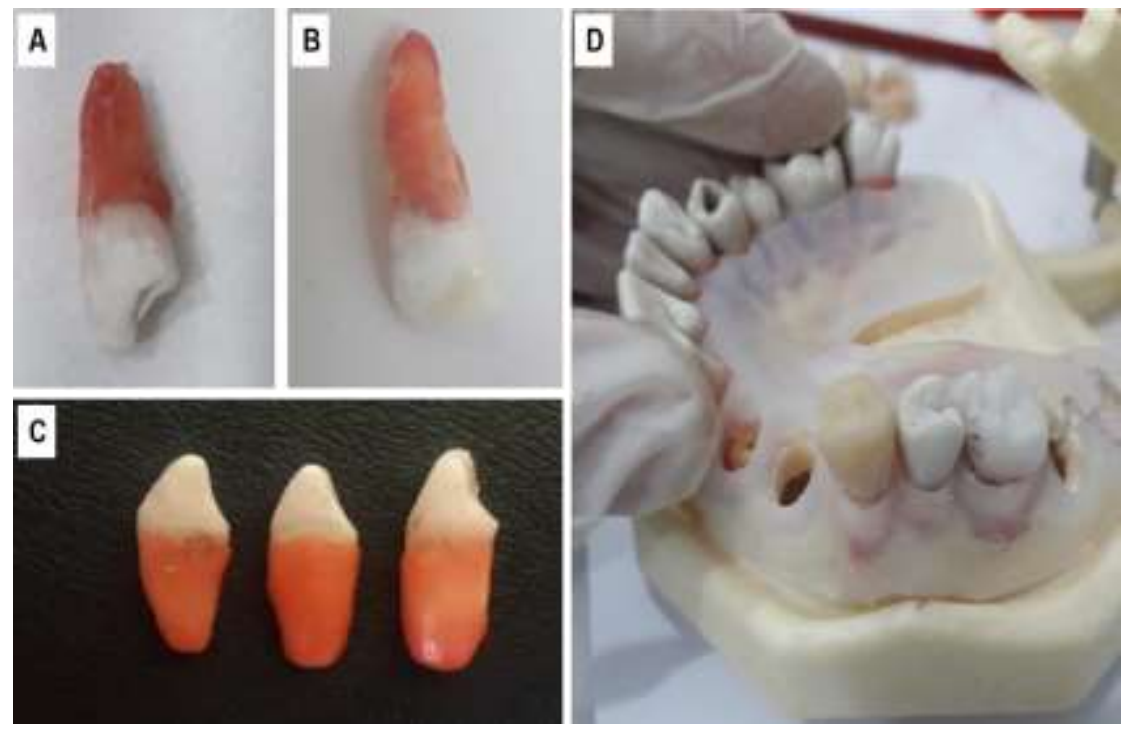

Fonte: Autoria própria. 
Para o tratamento endodôntico optou-se pela realização no dente 24 (Figura 4) pelo fato de possuírem duas raízes, visando a utilização da técnica de Clark, para ampliação do conhecimento radiográfico pré-clínico.

Figura 4. Radiografia da abertura (A), lima final posicionadas nos canais (B), cones principais posicionados no CRT para radiografia (C), radiografia dos cones principais no CRT(D), dente sendo obturado (E), radiografia após o corte dos cones (F).

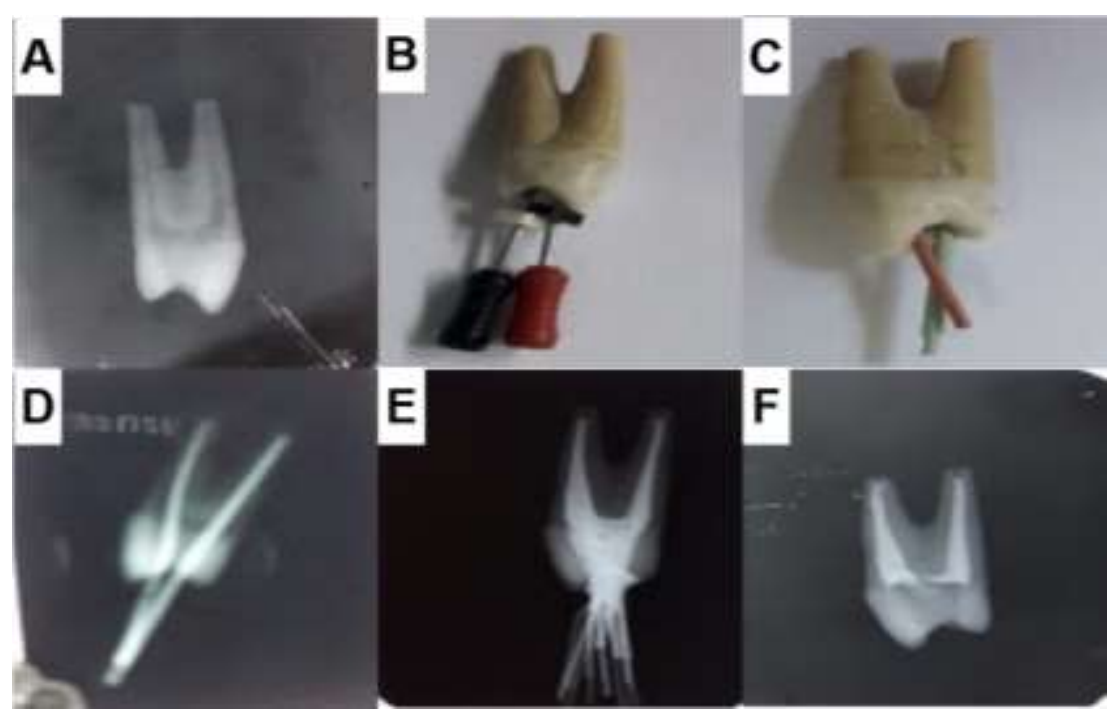

Fonte: Autoria própria.

O cisto residual foi confeccionado na região do dente 48, sendo confeccionado apenas com bolinha de cera 7 simulando a radiolucidez da lesão, ocasionada pela não curetagem do alvéolo.

A seguir, nas tabelas 1 e 2, estão especificadas todas as características do novo dispositivo didático-pedagógico para o ensino da radiologia odontológica pré-clínica com as formas de simulação de cada estrutura de forma simplificada, divididas em arcada superior e inferior.

Tabela 1. Características da arcada superior do novo dispositivo didático-pedagógico.

\begin{tabular}{lll}
\hline Dente & Característica & Simulação \\
11 & Restauração de resina classe IV & $\begin{array}{l}\text { Fratura da coroa com ponta diamantada 3216 e restauração } \\
\text { com resina composta. }\end{array}$ \\
\hline 12 & Raiz residual & Dente artificial removido a coroa. \\
& Dente colocado abaixo da gengiva de borracha na região do \\
13 & Incluso no palato & palato. \\
& Preparo de prótese fixa e & Dente artificial preparado com pontas diamantadas 3216 e \\
14 & provisório & 1014, e provisório com resina ativada quimicamente (pó e \\
& líquido). \\
\hline 15 & Hígido & Dente artificial radiopaco. \\
\hline 16 & Hígido & Dente artificial radiopaco. \\
\hline 18 & Ausente & Sem dente. \\
\hline 21 & Hígido & Dente artificial recoberto por gesso e maravalha. \\
\hline
\end{tabular}




\begin{tabular}{lll}
22 & Hígido & Dente artificial radiopaco. \\
23 & Hígido & Dente artificial radiopaco. \\
24 & $\begin{array}{l}\text { Tratamento endodôntico com } \\
\text { lesão apical }\end{array}$ & Dente artificial obturado com bolinha de cera 7 no ápice. \\
\hline 25 & Hígido & Dente artificial radiopaco. \\
& $\begin{array}{l}\text { Restauração de resina com } \\
\text { forramento com cimento de } \\
\text { hidróxido de cálcio. }\end{array}$ & Dente artificial com cavidade profunda restaurado de acordo \\
& Restauração de amálgama & Dente artificial com cavidade rasa restaurado por amálgama. \\
27 & Ausente & Sem dente. \\
\hline
\end{tabular}

Fonte: Autoria própria.

Tabela 2. Características da arcada inferior do novo dispositivo didático-pedagógico.

\begin{tabular}{|c|c|c|}
\hline Dente & Característica & Simulação \\
\hline 31 & Hígido & Dente artificial radiopaco. \\
\hline 32 & Hígido & Dente artificial radiopaco. \\
\hline 33 & Hígido & Dente artificial radiopaco \\
\hline 34 & Hígido & Dente artificial radiopaco. \\
\hline 35 & Raiz residual com lesão & $\begin{array}{l}\text { Dente artificial removido a coroa com esfera de cera } 7 \text { no } \\
\text { ápice da raiz. }\end{array}$ \\
\hline 36 & Implante com osseointegração & Implante com gesso e maravalha compactado ao redor. \\
\hline 37 & Hígido & Dente artificial radiopaco. \\
\hline 38 & Ausente & Sem dente. \\
\hline 41 & Hígido & Dente artificial radiopaco. \\
\hline 42 & Hígido & Dente artificial radiopaco. \\
\hline 43 & Hígido & Dente artificial radiopaco. \\
\hline 44 & Hipercementose & $\begin{array}{l}\text { Raiz do dente artificial sem ligamento periodontal (sem cera } \\
\text { 7) e colocação de camada de resina composta na raiz. }\end{array}$ \\
\hline 45 & Hígido & Dente artificial radiopaco. \\
\hline 46 & Implante sem osseointegração & $\begin{array}{l}\text { Implante com cera } 7 \text { ao redor simulando ausência de } \\
\text { osseointegração. }\end{array}$ \\
\hline 47 & Hígido & Dente artificial radiopaco. \\
\hline 48 & Cisto residual & Esfera de cera simulando radiolucidez. \\
\hline
\end{tabular}

Fonte: Autoria própria.

\section{Resultados}

O manequim foi acoplado ao simulador de paciente e adaptado a cadeira odontológica, com as técnicas de radiografia intrabucal, utilizando ou não os posicionadores, além de simular a posição do paciente e do feixe de raio X (Figura 5A). Durante os testes do manequim no simulador, utilizou-se o pregador de babador em cima do avental de chumbo, com objetivo 
de que o acadêmico aprenda que esse item deve ser removido antes das tomadas radiográficas (Figura 5B). Na Figura 5 é possível analisar o manequim finalizado já acoplado ao simulador de paciente.

Figura 5. Simulação de tomada radiográfica com posicionador de dentes posteriores inferiores do lado direito (A), pregador de babador em cima do avental de chumbo (B) e dispositivo finalizado com adaptações para simulação de paciente (C e D).

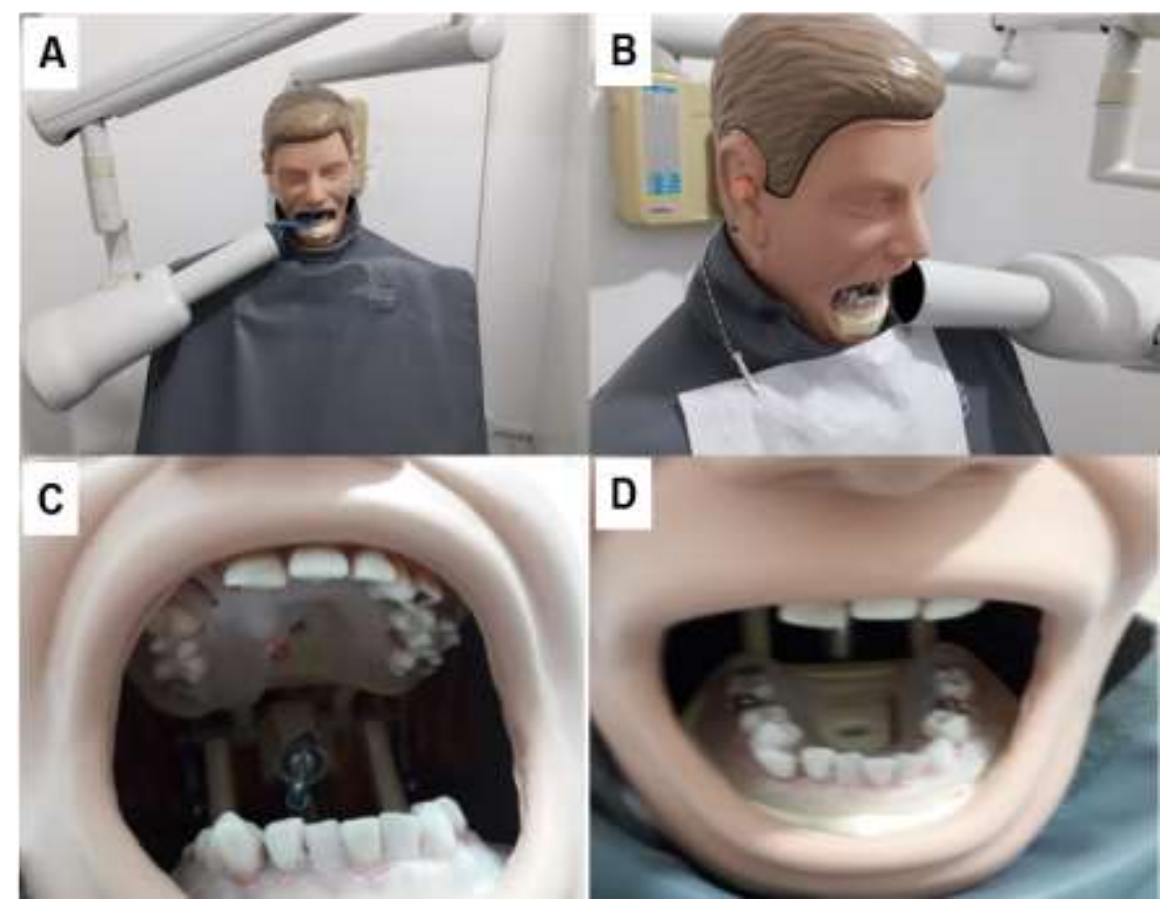

Fonte: Autoria própria.

Ao final da confecção do manequim, realizou-se radiografias periapicais de toda a cavidade bucal (Figura 6 A e B), sendo utilizado o tempo de exposição de $0.40 \mathrm{em}$ todas as tomadas radiográficas, evidenciando que é possível simular tanto as tomadas radiográficas em si, posicionamento correto dos filmes radiográficos, como as características ósseas dos dentes, tratamento endodôntico, restaurações, lesões apicais, raiz residual, implantes, dentes inclusos, preparo de prótese fixa e provisório, trabeculado ósseo, ausências dentárias e perdas ósseas.

Figura 6. Radiografias da arcada superior (A) e inferior (B).

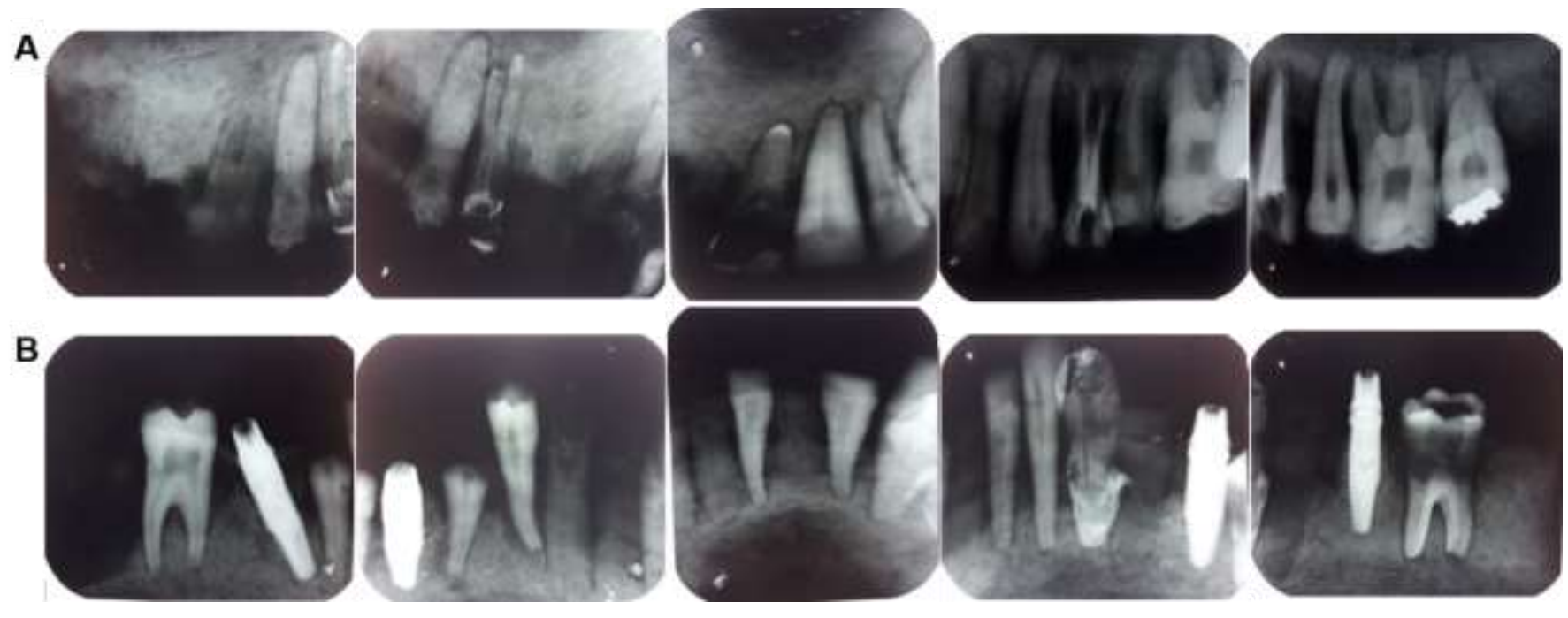

Fonte: Autoria própria. 
Na Figura 7 é possível analisar por meio de radiografia oclusal o dente 13 incluso no palato.

Figura 7. Radiografia oclusal evidenciando dente 13 incluso no palato.

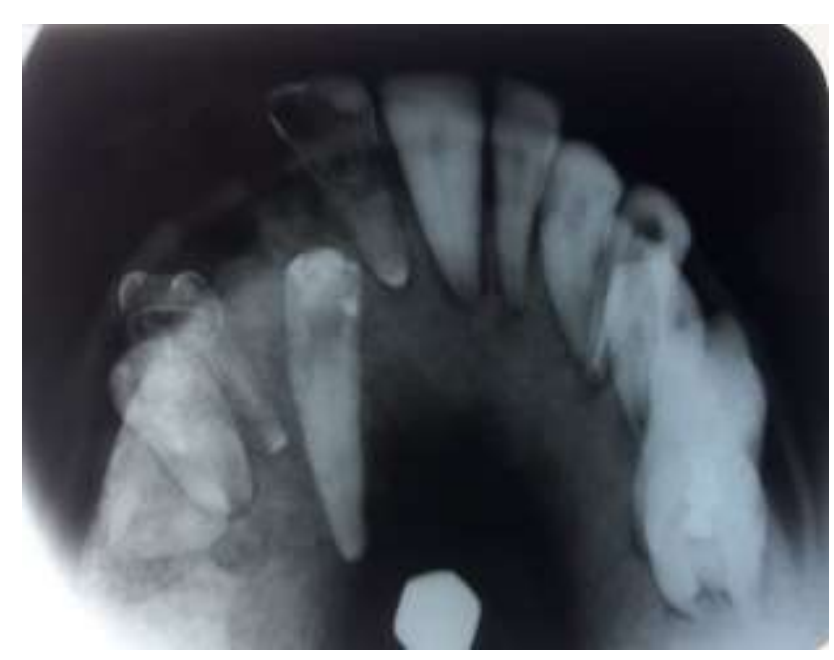

Fonte: Autoria própria.

\section{Discussão}

O ensino prático pré-clínico de Radiologia Odontológica representa uma importante etapa do aprendizado, pois permite aos estudantes a oportunidade de exercitar técnicas e construir a experiência necessária para posterior prática em pacientes (Luz et al., 2015). No entanto, o tradicional treinamento baseado em pacientes reais não é possível nas práticas atualmente, visto a contraindicação de exposição a radiações ionizantes sem necessidade (Gal et al., 2011; Tavano, 2000). Em contraste ao novo dispositivo produzido, a Figura 8 ilustra o modo que os acadêmicos de muitas faculdades realizam as tomadas radiográficas durante seu treinamento pré-clínico, o que dificulta o conhecimento e não possui limitações visuais, que costumam ocorrer na prática clínica.

Figura 8. Tradicional método de tomada radiográfica.

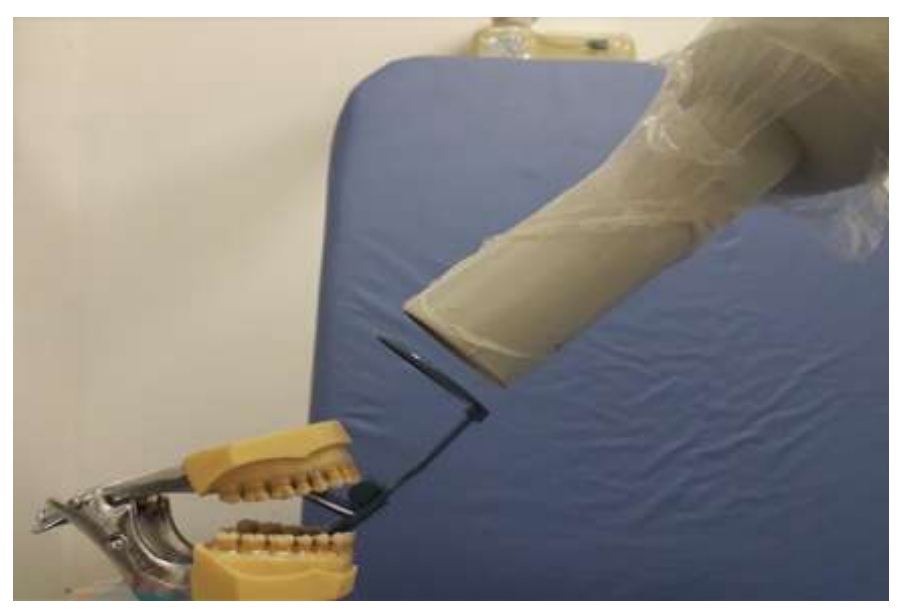

Fonte: Autoria própria.

Diferentemente do observado na Figura 8, o novo dispositivo desenvolvido possibilita que o aprendizado seja semelhante ao real, permitindo que o estudante possa praticar as angulações que $\mathrm{o}$ feixe de raios $\mathrm{x}$ deve estar sobre a face, pois 
o manequim pode ser acoplado a um simulador de paciente que fica fixado à cadeira odontológica (Figura 5). A não prática em pacientes se dá pelo fato de que apesar da radiação recebida ser muito pequena, os efeitos são cumulativos e capazes de gerar danos deletérios a longo prazo (Yarid et al., 2013; Garbin et al. 2015). Com isso, as preocupações com a segurança do paciente levaram a diminuição da popularidade da prática inicial em pacientes (LeBlanc et al., 2014; Wanzel et al., 2002; Arigbede et al., 2015).

Em alternativa ao uso de seres humanos (colegas de sala ou pacientes) para o treinamento das tomadas radiográficas, alguns cursos de Odontologia preconizam o uso de simulação e de ferramentas que permitem praticar e desenvolver essas competências (Gal et al., 2011), a fim de reproduzir ou imitar as condições clínicas reais (Luz et al., 2015). Além de aprendizado técnico para realização de radiografias, o novo dispositivo desenvolvido possibilita a interpretação de anomalias dentárias como dentes com restaurações de resina composta e amálgama demonstrando as classes I no elemento 26 e 27 e IV no dente 11, tratamento endodôntico no dente 24 , implantes, entre outros.

A possibilidade de colocação de isolamento absoluto no novo dispositivo ajuda o acadêmico quanto a visualização de como deverá ser o posicionamento e pontos de reparo que devem ser seguidos para a tomada radiográfica. O tratamento endodôntico do dente 24 proporciona o treinamento de radiografias pela técnica de Clark de dissociação das raízes.

No presente dispositivo, optou-se pelo uso de dentes artificiais no manequim, possuindo estes radiopacidade e radiolucidez semelhante a dentes naturais. O uso de dentes extraídos possibilita aos alunos a oportunidade de ganhar experiência antes de se mudar para os pacientes, entretanto, a infecção cruzada que pode ocorrer pela manipulação desses dentes e a falta de Biobanco de dentes humanos nas instituições de ensino, entre outras razões, tornam inapropriada tal prática (Nassri et al., 2008).

Quanto as características de radiopacidade dos materiais, Yamaji (2004) afirma que pó da lixa e a serragem são materiais de baixa densidade. Já o gesso apresenta particularidades que podem se alterar devido proporções de água. Sabe-se que os gessos apresentam uma expansão aparente do ponto de vista físico-químico, mas que se reflete na prática por um aumento de volume (Ferreira, 2000). No novo dispositivo o aspecto radiográfico de osso trabeculado é conseguido a partir da mistura de gesso e maravalha na proporção 2:1 (40g gesso tipo II e 20g maravalha).

A peça que acopla o simulador de paciente à cadeira odontológica (Figura 9) foi desenvolvida analisando o modelo da cadeira odontológica disponível para o estudo, encaixando-se de forma que o feixe de raio x pudesse ficar em uma altura compatível com a altura de um paciente normal. Ressalta-se que é possível criar uma peça semelhante de acordo com a cadeira odontológica disponível na instituição de ensino. Esse dispositivo juntamente com a articulação do manequim possibilita também a realização de radiografias oclusais. 
Figura 9. Peça que acopla o simulador de paciente a cadeira odontológica.

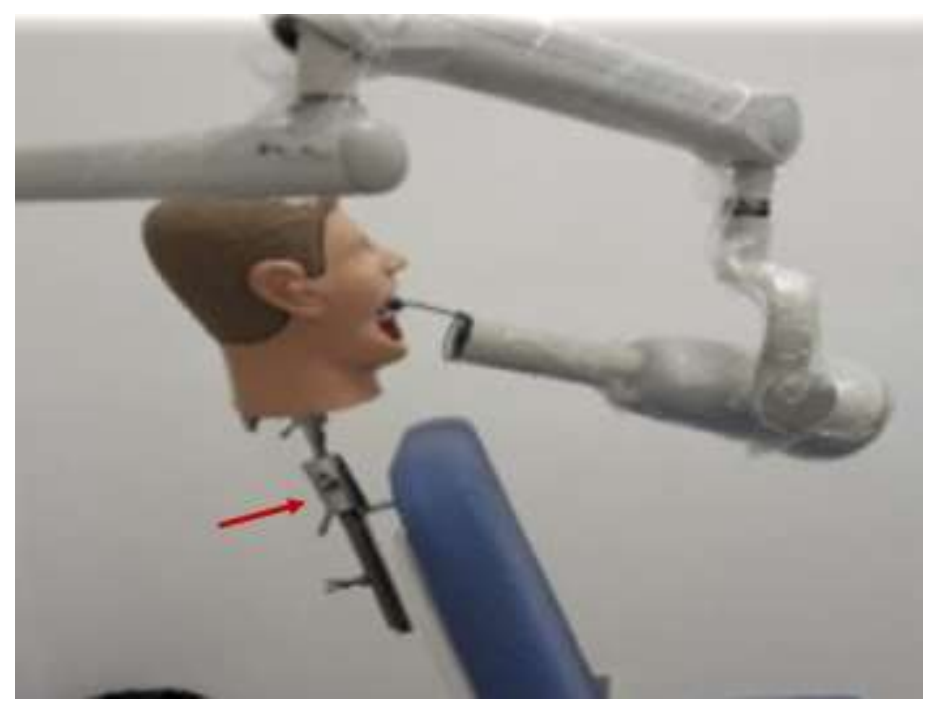

Fonte: Autoria própria.

Adicionalmente, o novo dispositivo possui características como perdas ósseas, espessamento do ligamento periodontal e anquilose. Além das características de multidisciplinaridade, existem as patologias, representadas no manequim por lesões apicais e cisto residual. Visto que é de extrema importância que o acadêmico saiba interpretar e analisar patologias comuns da cavidade oral, e nesse processo de aprendizado e descoberta sobre os benefícios da radiologia odontológica que se aprende a importância de diagnósticos de patologias que necessitam obrigatoriamente de exames radiográficos para o diagnóstico.

É válido salientar que o dispositivo pode ser confeccionado pelos próprios alunos podendo estes aprender e praticar durante a sua confecção, como preparos e restaurações de amálgama e resina composta, tratamentos endodônticos, classificação e posicionamento dos dentes inclusos, tanto de terceiro molares quanto no palato, e principalmente a possibilidade de realização de tomadas radiográficas, devido ao fato de os dentes possuírem características que simulam dentes naturais, e ter uma simulação de osso trabeculado que dá mais realidade e análise de como são visualmente as perdas ósseas e lesões mais comuns que podem acometer a cavidade bucal.

Essa metodologia ainda permite que seja utilizado adereços no simulador de paciente, como óculos, brincos, grampos, piercings, pregador de babador com o intuito do acadêmico aprender que esses itens devem ser removidos antes das tomadas radiográficas. Possibilita também o uso de avental de chumbo, para seguir uma correta biossegurança, servindo assim para abranger de forma mais ampla o ensino da radiologia odontológica.

De acordo com Lourenço et al. (2010), o exame radiográfico é amplamente utilizado na Odontologia, sendo altamente relevante visto que o diagnóstico radiográfico complementa o exame clínico e ajuda a resolver dúvidas diagnósticas, permitindo ao profissional planejar o tratamento com maior segurança por meio de análise das estruturas anatômicas além de patologias e anomalias.

$\mathrm{Na}$ especialidade Endodontia realiza-se muitas radiografias, e tanto o ensino laboratorial de Radiologia Odontológica quanto da Endodontia deixam a desejar no quesito tomadas radiográficas (Luz et al., 2015), o que dificulta a prática clínica e muitas vezes leva o aluno ou profissional a repetir as radiografias e, assim, repetir a exposição do paciente à radiações ionizantes. Sendo também válido para outras especialidades, como Periodontia, Dentística, Prótese, Implantodontia e Cirurgia, em que se recomenda o uso de radiografias tanto para a verificação da anatomia, quanto para o diagnóstico de doenças e acompanhamento do tratamento. A multidisciplinaridade dentro da Odontologia é de extrema importância, e durante a formação acadêmica é essencial que essa visão exista no acadêmico. Semelhante ao dispositivo desenvolvido neste estudo. 
É importante ressaltar que os materiais utilizados para a confecção do manequim são utilizados rotineiramente no dia a dia clínico de um ambiente odontológico, além de serem de baixo custo, fácil confecção e permite as tomadas radiográficas intraorais com perfeição. No entanto, uma sugestão para trabalhos futuros é a realização de uma pesquisa para comparar o aprendizado utilizando o novo dispositivo tanto na prática pré-clínica como se há melhora nas tomadas radiográficas nas práticas clínicas pós aprendizado com o novo dispositivo.

\section{Conclusão}

Conclui-se que é possível confeccionar um manequim odontológico que mimetize as características da cavidade bucal radiograficamente e que seja adequado para simular as tomadas radiográficas no ensino pré-clínico com baixo custo.

\section{Agradecimentos}

Agradecimento a CoPPExi do UNITPAC pelo apoio financeiro para a realização da pesquisa.

\section{Referências}

ADACSA (American Dental Association Council on Scientific Affairs). (2006). The use of dental radiographs: update and recommendations. The Journal of the American Dental Association, 137(9), 1304-1312.

Arigbede, A., Denloye, O., \& Dosumu, O. (2015). Use of simulators in operative dental education: experience in southern Nigeria. African health sciences, 15(1), 269-277.

Boeddinghaus, R., \& Whyte, A. (2008). Current concepts in maxillofacial imaging. European journal of radiology, 66(3), 396-418.

Capelozza, A. L. A., Alvares, L. C., Tavano, O., Freitas, J. A. D. S., \& Damante, J. H. (1993). Higiene das radiaçöes: radiologia preventiva. In Curso de radiologia em odontologia (pp. 41-50).

Ferreira, A. R., Nagem Filho, H., \& Pinto, J. H. N. (2000). Determinação da magnitude de expansão de alguns tipos de gesso. Salusvita, 19(2), 29-39.

Gal, G. B., Weiss, E. I., Gafni, N., \& Ziv, A. (2011). Preliminary assessment of faculty and student perception of a haptic virtual reality simulator for training dental manual dexterity. Journal of dental education, 75(4), 496-504.

Garbin, C. A. S., Wakayama, B., DE Lima, T. J. V., \& Garbin, A. J. Í. (2015). Condutas de proteção radiológica em odontologia: o que sabem os futuros profissionais?. Revista Uningá, 46(1).

Yarid, S. D., Nascimento, C. C., Alves, G. N., \& ALMEIDA, T. Y. L. (2013). Qualidade de vida de cirurgiões-dentistas da cidade de Jequié-Bahia. REVISTA UNINGÁ, 38(1).

Jackson, A. P., \& Tidmarsh, B. G. (1993). Simulation models for teaching endodonticsurgical procedures. International endodontic journal, $26(3)$, 198-200.

LeBlanc, VR, Urbankova, A., Hadavi, F., \& Lichtenthal, RM (2004). Um estudo preliminar sobre o uso de realidade virtual para treinar estudantes de odontologia. Journal of Dental Education , 68 (3), 378-383.

Lourenço, A. D. A., Pontual, A. D. A., Silveira, M. M. F. D., \& Pontual, M. L. D. A. (2010). Radiographic image quality after interruption of the fixing stage to view the image with a viewbox. Revista Odonto Ciência, 25, 78-82.

Luz, D. D. S., de S. Ourique, F., Scarparo, R. K., Vier-Pelisser, F. V., Morgental, R. D., Waltrick, S. B., \& de Figueiredo, J. A. (2015). Preparation time and perceptions of Brazilian specialists and dental students regarding simulated root canals for endodontic teaching: a preliminary study. Journal of dental education, 79(1), 56-63.

Nassri, M. R. G., Carlik, J., Silva, C. R. N. D., Okagawa, R. E., \& Lin, S. (2008). Critical analysis of artificial teeth for endodontic teaching. Journal of Applied Oral Science, 16(1), 43-49.

Del Queiroz, R. M., Botacin, W. G., Ortega, A. L., Leite, M. F., Pereira, T. C. R., \& de-Azevedo-Vaz, S. L. (2017). Análise da prescrição de radiografias por acadêmicos de Odontologia de uma universidade pública brasileira e desenvolvimento de um modelo didático. Revista da ABENO, $17(3)$, 100-109.

Ruprecht, A. (2009). The status of oral and maxillofacial radiology worldwide in 2007. Dentomaxillofacial Radiology, 38(2), 98-103.

Silva, A. C. A; Oliveira, E. B; Villibor, F. F.; Ribeiro, A. L. R (2018). Ensino prático de radiologia odontológica: desenvolvimento de uma nova metodologia. In: Jornada Odontológica ITPAC Araguaína. Anais... Araguaína. 
Suvinen, T. I., Messer, L. B., \& Franco, E. (1998). Clinical simulation in teaching preclinical dentistry. European Journal of Dental Education, 2(1), 25-32.

Tavano, O. (2000). O máximo de segurança e qualidade na obtenção de radiografias odontológicas com um equipamento de $70 \mathrm{kV}$. Revista ABRO, Bauru, 1(1), 35-40.

Yamaji, F. M., \& Bonduelle, A. (2004). Utilização da serragem na produção de compósitos plástico-madeira. Floresta, 34(1).

Wanzel, K. R., Ward, M., \& Reznick, R. K. (2002). Teaching the surgical craft: from selection to certification. Current problems in surgery, 39(6), 583-659. 